\title{
Análise de dados qualitativos nas pesquisas sobre formação de professores
}

\author{
Qualitative data analysis in research on teacher's \\ education
}

\section{Análisis cualitativo de datos en la investigación sobre la formación del profesorado}

\section{Dilmeire Sant'Anna Ramos Vosgerau ${ }^{[a]}$, Patrícia Meyer ${ }^{[b]}$, Ricardo Contreras ${ }^{[c] *}$}

[a] Pontifícia Universidade Católica do Paraná (PUCPR), Curitiba, PR, Brasil

[b] Instituto Federal do Paraná Campus Curitiba (IFPR), Curitiba, PR, Brasil

[c] Universidade do Chile, Santiago, Chile

\section{Resumo}

Este artigo é fruto da palestra proferida no XII Congresso Nacional de Educação - EDUCERE na qual relatamos o processo de apropriação do software ATLAS.ti por estudantes de Pósgraduação na condução do processo de análise de dados de suas pesquisas, apontando

* DSRV: Doutora em Educação, e-mail: dilmeire.vosgerau@pucpr.br PM: Doutoranda em Educação, e-mail: patricia.meyer@ifpr.edu.br RC: Doutor em Antropologia, e-mail: ricardo.contreras@atlasti.com 
os desafios transpostos por eles, por meio da retomada das pesquisas qualitativas empreendidas. Em comum, os estudantes participaram da disciplina de "Análise de dados qualitativos utilizando recursos tecnológicos", na PUCPR, ministrada desde 2004, ou de oficinas oferecidas em diversas universidades, nas quais são instrumentalizados para o uso do software ATLAS.ti. Apresentamos suas experiências reais, contextualizadas com os principais recursos disponibilizados pelo software e orientamos sobre a necessidade de capacitar os pesquisadores qualitativos para a realização de um consistente plano de análise de dados, que possa integrar as questões e subquestões de investigação propostas em sua pesquisa. Destacamos que a não definição de um plano influi na desistência de uso do software com muito mais frequência que possíveis barreiras tecnológicas. A partir da organização do plano, a aplicação das alternativas disponíveis pelo software é facilitada o que contribui para resultados mais significativos e consistentes na análise de dados.

Palavras-chave: Análise de dados qualitativos. ATLAS.ti. Formação de professores.

\section{Abstract}

This article is the result of a paper presented at the XII National Congress on Education (EDUCERE), in which we discussed the process of appropriation of the ATLAS.ti software by graduate students engaged in qualitative data analyses processes. The graduate students on which the article is based had enrolled in the class 'Analysis of qualitative data using technological resources', which has been offered at the Catholic University of Paraná (PUCPR) since 2004, as well as on students who took equivalent courses in other universities, all of them using ATLAS.ti. In the article, we present experiences related to their usage of the software. We looked at the need to provide training to students on qualitative data analysis as methodology. We make the point that lack of methodological expertise, rather than technological barriers, shapes some of the participants' decision not to continue using the software. We also notice the use of the software is facilitated by having knowledge and expertise on qualitative methodology.

Keywords: Qualitative data analysis. ATLAS.ti. Teacher training. 


\section{Resumen}

Este articulo es el producto de la ponencia presentada en el XII Congreso Nacional de Educación (EDUCERE), en el cual se presentó respecto al proceso de apropiación del software ATLAS.ti de parte de estudiantes participantes del curso 'Análisis cualitativo de datos usando recursos tecnológicos, el cual se ha estado ofreciendo en la Universidad Católica de Paraná (PUCPR) desde el año 2004. En este articulo presentamos las experiencias de los alumnos con el software y señalamos la importancia de que la enseñanza del software vaya acompañado con la enseñanza de metodología cualitativa. En ese sentido, presentamos la tesis de que la falta de conocimientos metodológicos es la principal razón que explica la decisión de algunos estudiantes de no persistir en el uso del software. Al mismo tiempo, señalamos que poseer conocimientos de metodología facilita el aprendizaje del software.

Palabras clave: Análisis de datos cualitativos. ATLAS.ti. Formación de profesores.

\section{Introdução}

A pesquisa qualitativa é uma atividade situada que localiza o observador no mundo, se compõe por um conjunto de práticas interpretativas e materiais que tornam o mundo visível, transformando-o em uma série de representações, incluindo notas de campo, entrevistas, conversas, fotos, gravações e memorandos. Envolve, portanto, uma abordagem interpretativa, naturalista ao mundo. Isso significa que os pesquisadores qualitativos estudam as coisas em seus ambientes naturais, tentando dar sentido ou interpretar fenômenos nos termos das significações que as pessoas trazem para eles (DENSIN; LINCOLN, 2011).

Essas pesquisas têm como características: o cenário natural, o pesquisador como instrumento fundamental da coleta de dados, o uso de métodos múltiplos, um design que emerge em cada projeto de pesquisa empreendido, foco no significado para os participantes, reflexiva e raciocínio complexo por meio da perspectiva holística (CRESWELL, 2013). 
Com essa multiplicidade, complexidade de características e seu avanço crescente, os pesquisadores qualitativos se vêm confrontados com diversos desafios.

Primeiro, surge o apelo de diferentes revistas científicas para que, não apenas, seja derrubada a divisão quantitativa/qualitativa (ERCIKAN; ROTH, 2006), mas para que ambos os tipos de estudos sejam complementares e atuem em um movimento circular, podendo seus resultados serem cruzados, compartilhados e complementados um pelo outro (CRESWELL, 2003).

Segundo, outro desafio se refere à possibilidade de utilização de uma diversidade cada vez maior e não previsível de fontes de dados nas pesquisas qualitativas (LEEDY; ORMROD, 2001). Enquanto nas pesquisas quantitativas os dados já são coletados de forma sistematizada, normalmente com instrumentos fechados ou surveys, nas pesquisas qualitativas além das fontes de origem textual, tais como, entrevistas abertas, semiestruturadas, análises documentais, roteiros de observação e relatos autobiográficos, surge a possibilidade de utilização de imagens, áudios, vídeos e ainda os dados virtuais, gerados por meio de fóruns, blogs e redes sociais. Soma-se a isso a necessidade de articular, associar e discutir essas informações com resultados quantitativos.

Consequentemente urge ao pesquisador qualitativo instrumentalizar-se em face desses desafios. O processo de pesquisa por meio da bricolagem já não é mais suficiente (WALTER; BACH, 2015), assim como a descrição individualizada de trechos das falas dos sujeitos nos relatórios de pesquisa. De forma individualizada são infrequentes os cruzamentos dos indicadores levantados pela pesquisa com dados demográficos que poderiam explicar particularidades e especificidades da situação encontrada. Também é raro encontrarmos a descoberta de coocorrências dos indicadores levantados na codificação, e a geração de redes conceituais que nos permitam compreender as nuances e articulações presentes em um resultado qualitativo. É fato que, no contexto de complexidade social em que vivemos, precisamos avançar e buscar novos instrumentos que nos 
auxiliem a ter uma compreensão mais ampla e, simultaneamente, mais profunda dos resultados de pesquisa qualitativamente descritos.

Questionamentos sobre a necessidade, ou não, de mais instrumentos para a pesquisa qualitativa datam o início dos anos de 1990, quando Tesch (1990) apontava alguns mitos que estavam presentes na tomada de decisão sobre a utilização, ou não, de softwares para pesquisas qualitativas. Mesmo com a evolução dos softwares, há mitos que ainda se fazem presentes. Um dos principais, assinalados por Tesch (1990), e que alguns pesquisadores discretamente manifestam, é o receio de que o software determinaria a condução do processo de análise de dados. Se isso ocorresse de fato, não teríamos inúmeros trabalhos conduzidos em uma mesma área ou em áreas diferentes com o mesmo software, utilizando encaminhamentos metodológicos e procedimentos analíticos tão distintos.

Uma das conclusões de Tesch (1990), e que permanece válida até hoje, é que o conhecimento sobre o objeto de estudo, sobre o método de análise de dados escolhido e a criatividade do pesquisador é que garantirão um uso eficiente do software. Portanto, seria fundamental ao pesquisador tomar decisões sobre seu processo de análise de dados, logo, planejá-lo e conhecê-lo profundamente antes de se aventurar na escolha ou no uso de um software específico (MACMILLAN; KOENIG, 2004).

Esse entendimento nos motivou a conduzir este trabalho apresentado no XII EDUCERE, no qual estabelecemos como objetivo identificar as apropriações do software feitas pelos estudantes na condução do seu processo de análise de dados e os desafios transpostos por eles para essa apropriação.

Para isso, resgatamos pesquisas realizadas por estudantes de Pós-graduação que participaram da disciplina "Análise de dados qualitativos utilizando recursos tecnológicos", na PUCPR, desde 2004, na qual o uso do software ATLAS.ti era associado à técnica de análise de conteúdo proposta por Bardin (1977; 2006; 2009) e outros casos, de estudantes que participaram de formação para uso do mesmo software por meio de oficinas oferecidas em diversas universidades brasileiras. Trazemos ainda à tona a experiência de quatro ex-alunos de diferentes programas de 
Pós-graduação ${ }^{1}$ que utilizaram o software ATLAS.ti em suas pesquisas, aos quais agradecemos formalmente a nobreza em compartilhar com os colegas os anseios, desafios e conquistas nessa etapa de formação como pesquisadores.

Para discutir o processo de apropriação, primeiramente apresentamos algumas alternativas no processo de tratamento de dados qualitativos utilizando o software ATLAS.ti. Na sequência, buscamos relacionar os limites e possibilidades de utilização apresentados nas dissertações e teses produzidas pelos estudantes, às alternativas disponíveis pelo software.

\section{O processo de análise de dados qualitativos utilizando o ATLAS.ti}

A análise na pesquisa qualitativa consiste inicialmente na preparação e organização dos dados (logo, textos transcritos, ou imagens, como vídeos e fotografias), para então proceder-se à redução dos dados a temas por meio de um processo de codificação e condensação dos códigos, para finalmente apresentar sua representação em figuras, tabelas, ou em discussão (CRESWELL, 2013).

Contudo, a análise de dados qualitativa pode se iniciar já durante a coleta de dados (MILES; HUBERMAN, 1994), quando o pesquisador ao estar em campo acompanhando, observando ou entrevistando constitui seu diário de bordo (MACHADO, 2016). Esse diário poderá compor o corpus de análise e nele conterão informações e reflexões únicas que poderão ser confrontadas ou enriquecer a análise do material coletado.

Ao descrever o processo de análise via grounded theory ou teoria fundamentada nos dados, Tarozzi (2011) compara a utilização de diário de pesquisa (ou de bordo) com os memorandos (memos). A preocupação

\footnotetext{
Agradeço a participação, contribuição e generosidade dos seguintes colegas em partilhar conosco suas experiências com a utilização do ATLAS.ti: Dr. Jacques de Lima Ferreira (ex-aluno do programa de pós-gruaduação da PUCPR); Doutorandas Michele Simonian (UFPR) e Dagmar Heil Procrifka (UFPE); Mestre Genaldo Luis Sievert (ex-aluno do programa de pós-graduação da PUCPR); e Mestranda Marina Lupepso (PUCPR).
} 
do autor, em realizar a diferenciação, está no fato de que ao analisar os dados por meio da grounded theory, o pesquisador qualitativo realiza simultaneamente a coleta e a análise de dados, empreendendo a codificação e categorização em uma perspectiva espiral e não linear, até a saturação teórica e a emersão de uma teoria — por isso a denominação de ancorada ou fundamentada nos dados. O processo de análise é permeado pelo uso de memorandos e também do diário de pesquisa, com funções distintas. Ao primeiro cabe registrar o que "não é óbvio", os questionamentos, as justificativas e a argumentação que se constroem no e a partir do processo de decisão do pesquisador. Os memorandos são para enfatizar trechos essenciais, destacar as articulações e associações fundamentais para a realização da análise e prestar contas "das perguntas que se fazem aos dados e às categorias e das razões em virtude das quais são dadas apropriadas respostas às mesmas" (TAROZZI, 2011, p. 76). Embora existam momentos nos quais o uso possa coincidir, ao diário de pesquisa cabe mais um acompanhamento pessoal da pesquisa, com autorreflexão e registros que enriqueçam a análise, mas não a constituam em si.

Verifica-se, então, que diferentes estratégias podem ser utilizadas no processo de análise de dados qualitativa, tais como: esboçar ideia; tomar notas; registrar palavras-chaves; identificar códigos; identificar temas; contar palavras; contar frequências de códigos; relacionar categorias; relacionar categoria a um quadro teórico; criar pontos de vistas; e elaborar esquemas de visualização de dados.

Atualmente, diferentes softwares oferecem ferramentas que podem auxiliar o pesquisador na aplicação dessas e outras estratégias, assim como na gestão de seus resultados.

O Quadro 1 evidencia que, embora os softwares de análise de dados disponham de diferentes funcionalidades que podem favorecer a qualidade e o rigor científico no tratamento de dados, são as atribuições do pesquisador que efetivamente são essenciais na interpretação e representação na pesquisa qualitativa. As diversas formas de aplicação do software e sua potencialidade em lidar com grandes volumes de dados são úteis e 
importantes. Porém, reforçam o quanto é fundamental ao pesquisador desenvolver um plano de análise de dados, coerente com seu problema de pesquisa e que permita a melhor exploração, não apenas dos dados, mas também do suporte oferecido pelo programa.

Quadro 1: Relação entre pesquisador e software de análise de dados qualitativos

Atribuições do Pesquisador

Atividades relativas ao software de análise de dados

Criação e organização dos arquivos com os dados

Leitura dos dados e das anotações registradas na etapa de coleta visando uma primeira análise

Início da codificação, a partir do plano de análise de dados determinado pelo objetivo de pesquisa

Classificação e categorização dos dados, estabelecendo relações e conexão entre os dados, entre os dados e os perfis dos sujeitos pesquisados, com os conceitos teóricos
Atua como banco de dados das informações e documentos de pesquisa.

Possibilita fácil acesso, busca e resgate de dados, sejam eles arquivos de texto, áudio, fotos ou vídeos. Facilita um exame minucioso.

Recupera anotações (memos) realizadas ao longo do percurso.

Permite relacionar e associar os dados, gerando relatórios e imagens (facilitando comparações), assim como frequências de palavras, por exemplo. Dá suporte a apresentação visual dos dados.

Fonte: as autoras, a partir de CRESWELL (2013)

Entre os softwares de análise de dados qualitativos está o ATLAS. ti, que iniciou seu processo de comercialização em 1993 e tem sua fonte de desenvolvimento localizada em Berlin-Alemanha.

O software ATLAS.ti facilita o processo de análise de dados qualitativos e ajuda a conduzir um processo de análise fundamentado em evidências, além de possibilitar a recuperação dos caminhos de análise percorrido pelo pesquisador (CONTRERAS, 2015). Também auxilia a organizar e manter um banco de dados tanto dos dados brutos (por instrumento de coleta) como dos dados sistematizados visualmente (por sujeitos ou categorizados em relação aos objetivos de pesquisa), o que permitem um acesso mais ágil no momento de análise (MEYER, 2010).

O trabalho no interior do software é interativo, por meio da oferta ao pesquisador de ferramentas para descrição, análise e interpretação dos dados que permitirão produzir uma representação holística 
fundamentada nas evidências. Isso transcorre por meio dos processos de: segmentação de dados, codificação, escrita e diagramação (Figura 1). Contudo, antes da execução desses processos se dá a preparação dos dados para que se constitua o corpus da pesquisa.

\section{Figura 1 - Processo interativo para análise de dados no ATLAS.ti}

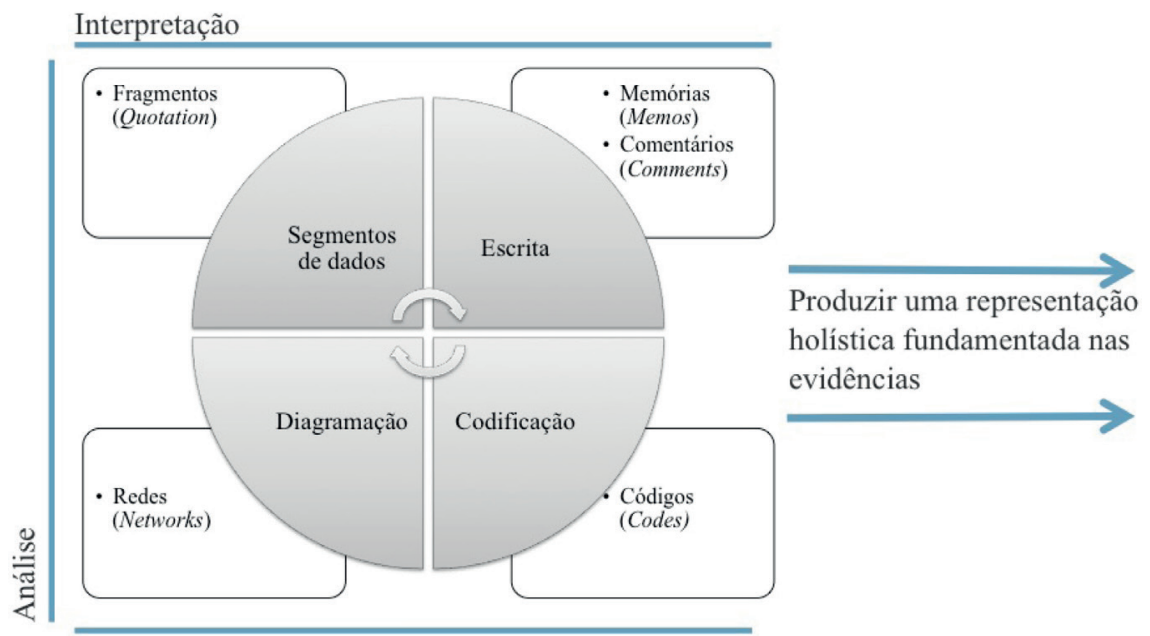

Descrição

Fonte: Contreras (2015).

A constituição do corpus se inicia quando o pesquisador começa a organizar fisicamente o material coletado para facilitar o acesso aos documentos, seja por ordem de data de coleta, tipos de fontes, momentos específicos da coleta ou outras formas. Essa organização se dá virtualmente, e começa pela nomenclatura atribuída a cada documento, podendo ser um arquivo de texto do Word (.doc ou .txt), imagens (.jpg), arquivos no formato .pdf, entre outros, visto que ao utilizar o ATLAS.ti este nome pode ser associado aos extratos do documento analisado, permitindo ao leitor conhecer o documento de origem do fragmento ${ }^{2}$ selecionado na análise.

2 Utilizamos a palavra Fragmento para tradução de Quotation. Segundo o dicionário Aurélio de 
Outra decisão a ser tomada é quanto ao conteúdo que será inserido em cada arquivo, por exemplo: questionários quando preenchidos em documento Word normalmente são individualizados e inseridos no software em arquivos separados (FERREIRA, 2015); porém, quando são elaborados por ferramentas específicas para realização de surveys, tais como Qualtrics ou Google Forms, geram arquivos no formato Excel, nos quais, cada linha corresponde a um sujeito e cada coluna a uma questão, podendo ser importados pelo ATLAS.ti como survey sendo cada linha tratada como um participante individual. Para entrevistas, o pesquisador terá de tomar a decisão se serão transcritas na íntegra e inseridas em arquivo de texto individualmente ou se serão analisadas por áudio e transcritos apenas os estratos selecionados durante a análise dos dados. Para grupos focais e fóruns de discussão (BUCHI, 2006; SILVA, 2010; SIEVERT, 2013) ou em mensagens trocadas em redes sociais por um grupo (ex. Twitter, Facebook), as discussões são normalmente inseridas em um único arquivo, pois, normalmente deseja-se conhecer o processo de interação ocorrido nas trocas de mensagens.

Buchi (2006) realizou esse tipo de análise com a versão 4.1 do software, o que gerou um trabalho maior de preparação de dados copiando e colando cada mensagem trocada entre os participantes da pesquisa para um documento Word, retirando caracteres especiais e tabulações que pudessem comprometer a visualização dos dados dentro do software. Silva (2010), que já utilizava a versão 5.0, conseguiu extrair todas as mensagens uma única vez do ambiente virtual no qual foi realizado o fórum, ordenando-as alfabeticamente por participante, pois seu problema de pesquisa não se relacionava às interações. Sievert (2013) ao utilizar a versão 7.0 pode copiar as informações do ambiente ordenadas tematicamente, guardando a ordem e interação entre os participantes.

Por esses exemplos, observa-se que, já nessa fase, é essencial o planejamento da organização virtual das informações recolhidas visando

língua Portuguesa (FERREIRA, 2009), a palavra Fragmento significa "Parte de um todo; pedaço ou fração", portanto ela representa adequadamente a multiplicidade de tipos de frações que podem ser analisadas dentro do Atlas.ti: partes de texto, áudio, vídeo e imagem. 
atender os objetivos propostos pela pesquisa, caso contrário após a análise o pesquisador não conseguirá fazer os questionamentos necessários para obter do software os cruzamentos esperados. Além desses cuidados, para uma melhor organização da pesquisa é recomendável que seja criada uma pasta que abrigará todos os documentos relacionados ao projeto de pesquisa.

Após essa organização virtual do material a ser analisado, o projeto é criado dentro do software ATLAS.ti, denominado pelo software como Hermeneutic Unit. Ressalta-se que um projeto, ou uma Hermeneutic Unit, deve conter todo o Corpus de análise da pesquisa, todos os documentos que compõem o processo de análise de dados e auxiliarão a responder a questão de pesquisa proposta.

O ATLAS.ti oferece seis objetos (Figura 2) essenciais na fase que Saldaña (2016) denomina de $1^{\circ}$. Ciclo de Codificação em um projeto de análise de dados qualitativos: documentos primários; excertos; códigos; memórias; redes e famílias. Esses objetos coexistem em relação uns aos outros e estão integrados a uma unidade hermenêutica (projeto).

Figura 2 - Objetos disponíveis no ATLAS.ti

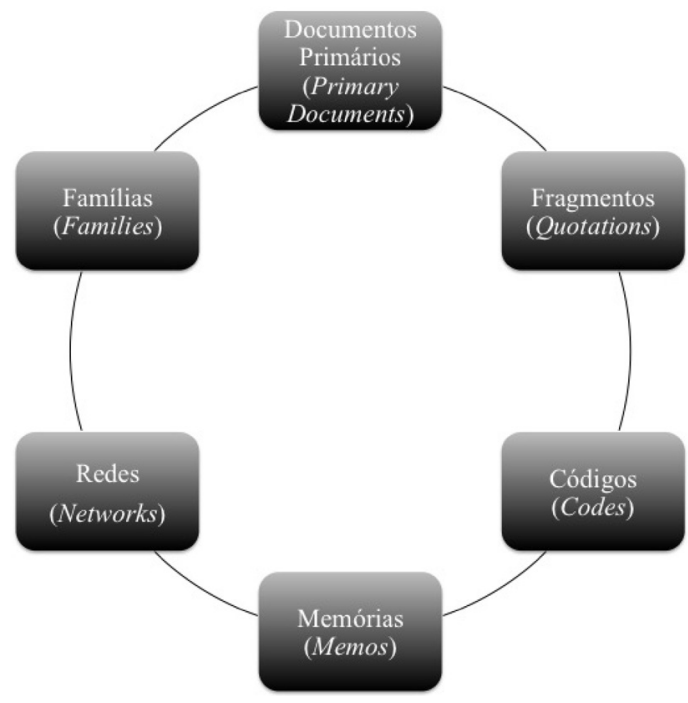

Fonte: Contreras (2015). 
Os documentos primários (Figura 3) são as fontes de dados coletadas no campo de pesquisa, sejam elas questionários, entrevistas, imagens, vídeos e fontes oriundas do Google Earth, não tendo uma limitação de tamanho.

Figura 3 - Exemplos de documentos primários - primary documents

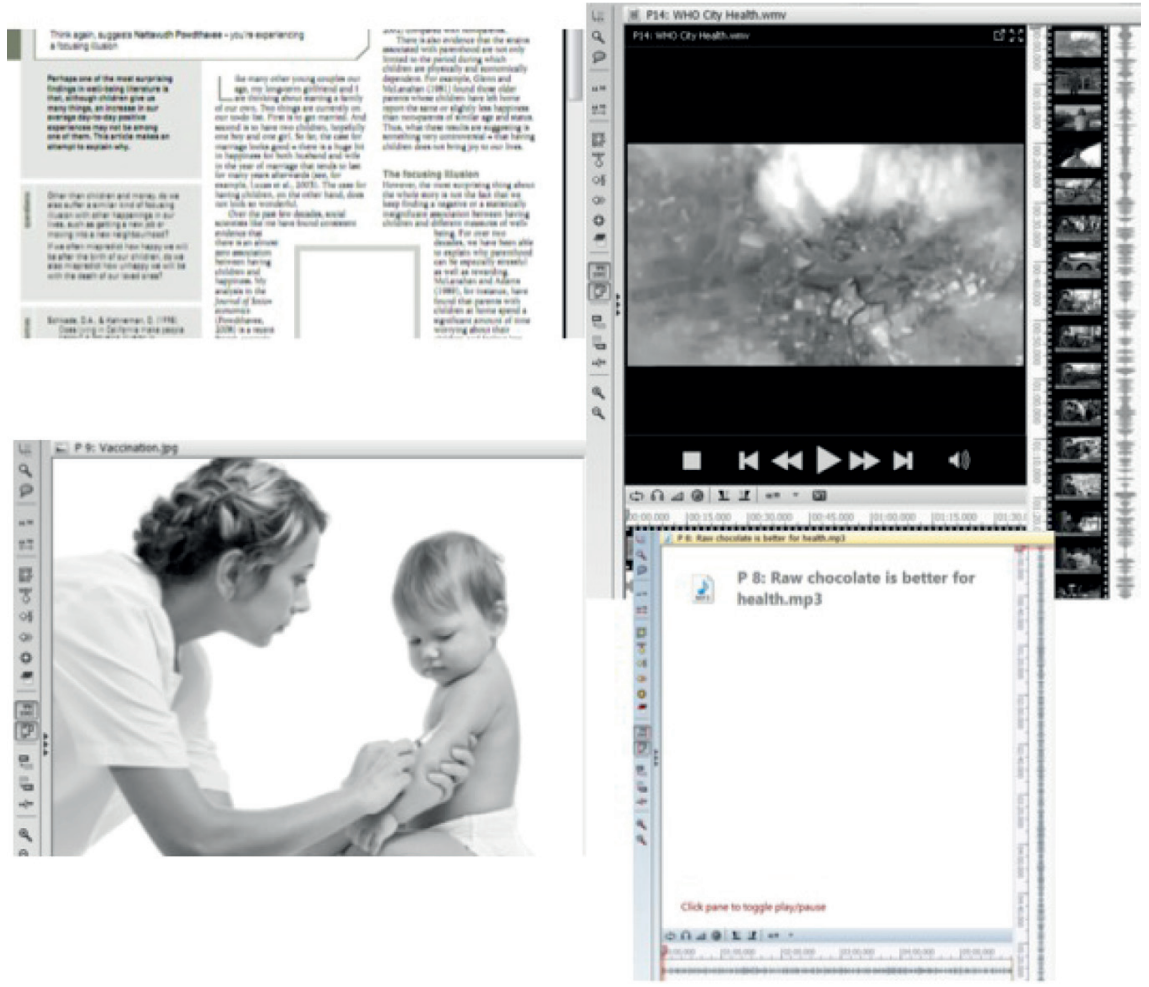

Fonte: Contreras (2015).

Os fragmentos (Figura 4) são os segmentos de texto, vídeo ou áudio, ou ainda, partes de imagem, de qualquer tamanho que contém algo significativo que possa auxiliar o pesquisador a responder a questão de pesquisa proposta. Eles são objetos independentes e podem, ou não, estar 
associados a códigos, como também podem estar relacionados entre si no mesmo documento ou entre documentos.

Figura 4 - Exemplos de fragmentos - quotation
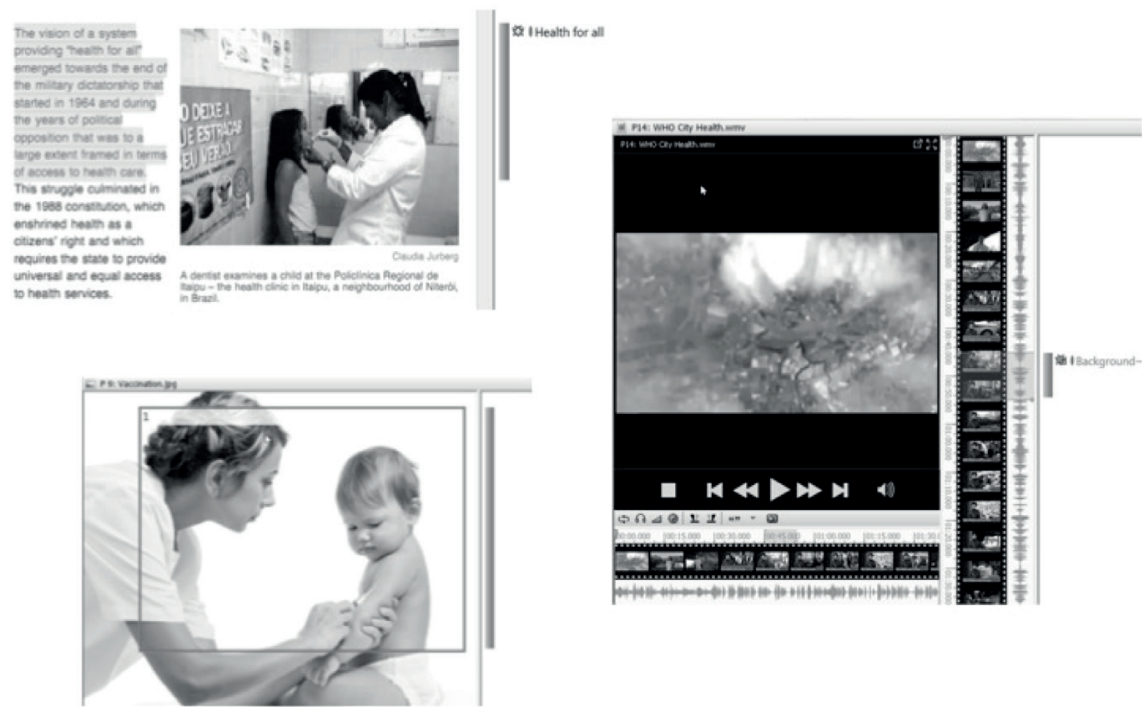

Fonte: Contreras (2015).

Os códigos (Figura 5) correspondem a uma denominação atribuída pelo pesquisador aos segmentos que possuem características em comum. Saldaña (2016, p. 3) os define como "palavra ou frase curta que simbolicamente atribui, salienta a essência de captura e/ou atributo evocativo para uma porção de dados baseada em linguagem ou visualmente". Eles normalmente são associados aos fragmentos, mas não obrigatoriamente. Também podem ser agrupados a outros e representados semanticamente. 
Figura 5 - Exemplo de códigos - codes

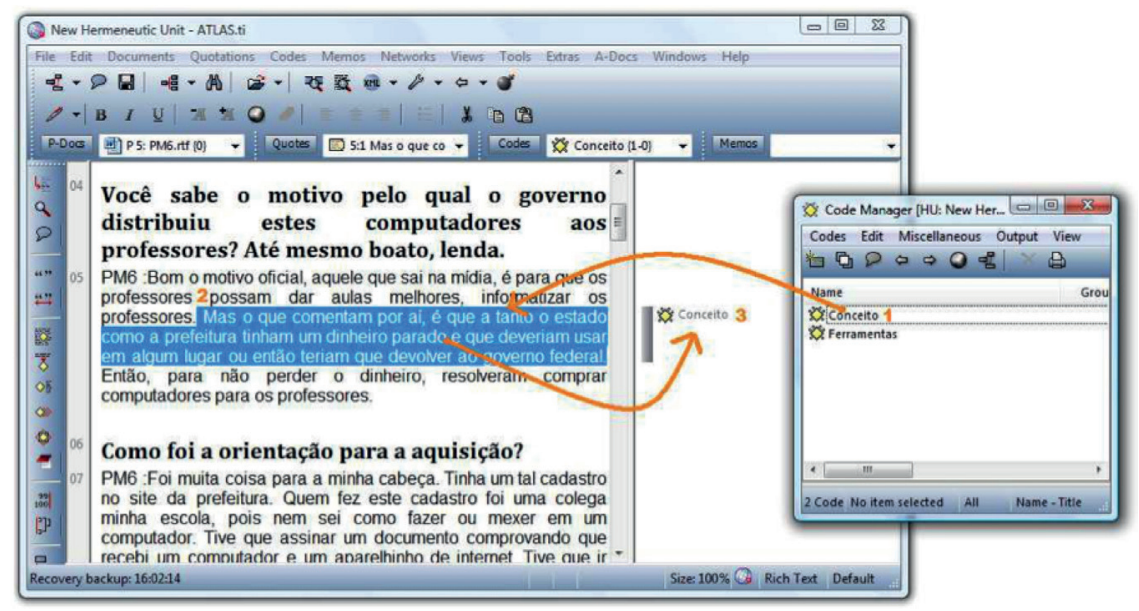

Fonte: Pocrifka (2012, p. 163).

As memos - memórias (Figura 6) são anotações realizadas pelo pesquisador que o ajudarão a compreender o excerto selecionado ou associações feitas relativas a alguma teoria que possa explicar o que foi selecionado, ou ainda qualquer ordem de anotação que possa contribuir para as inferências que serão feitas após a codificação e análise. Para Saldaña (2016, p. 44) "as memos analíticas são de alguma forma comparável a entradas de diário da pesquisa ou blogs, um lugar para 'Despejar seu cérebro'”. É, portanto, um espaço de integração, para reflexão e busca de sentido. Podem estar ou não associadas aos fragmentos, códigos ou outras memos. 
Figura 6 - Exemplos de memos

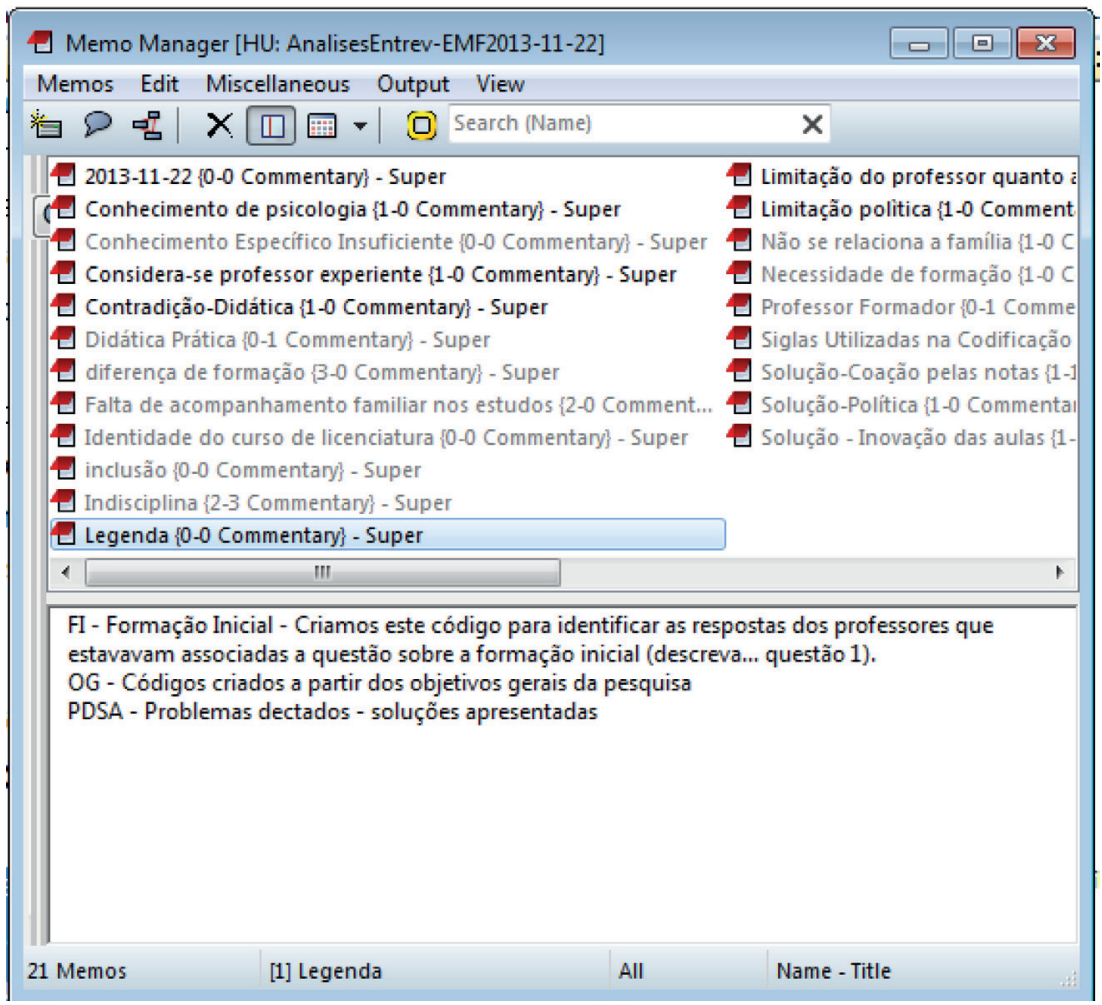

Fonte: acervo pessoal (2011).

O processo de análise de dados inicia-se pela leitura flutuante e exploração dos documentos inseridos no projeto (BARDIN, 2009) extraindo as unidades de sentido do texto. Essas unidades se referem aos fragmentos que contém informações que possam contribuir para responder a questão de pesquisa. Saldanã (2016) sugere um processo de criação de free quotations ${ }^{3}$ podendo ser nomeadas pelo seu conteúdo e ainda

3 Quotations são os extratos selecionados pelo pesquisador dentro do texto que está sendo analisado. 
comentadas utilizando o recurso de criação de memos existente no software. Essas memórias são fundamentais para que se inicie a compreensão dos dados da pesquisa e de algumas ideias que surgem na primeira leitura. Também podem ser questões a serem confirmadas em uma análise mais profunda.

As redes ou networks (Figura 7) são representações gráfica das associações (links) construídos pelo pesquisador durante a análise de dados. Podem ser feitas associações sem definição de tipo e associações tipificada, definidas como redes semânticas. Elas permitem ao pesquisador verificar como as peças se encaixam, ou ainda a elaboração de mapas conceituais e taxonomias.

Figura 7 - Exemplo de rede - network

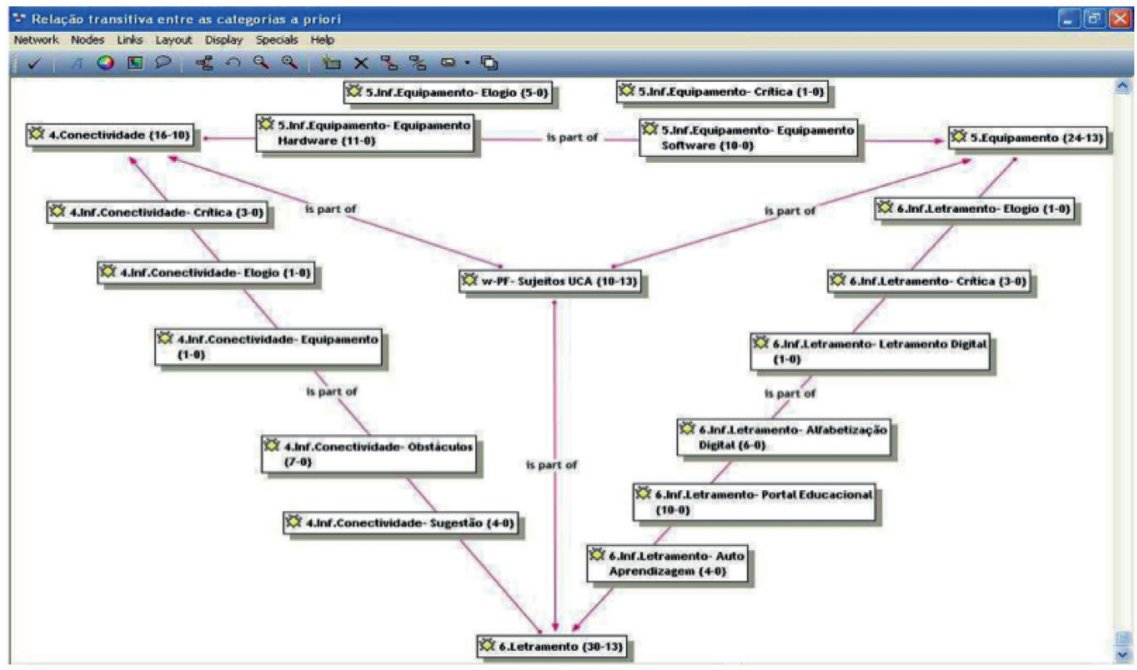

Fonte: Pofcrifka (2012, p. 98).

As famílias (Figura 8) são agrupamentos de documentos, códigos ou memos, de acordo com atributos e características compartilhadas e que vão permitir ao pesquisador realizar os questionamentos e cruzamentos necessários dos casos e domínios conceituais. 
Figura 8 - Exemplo de famílias

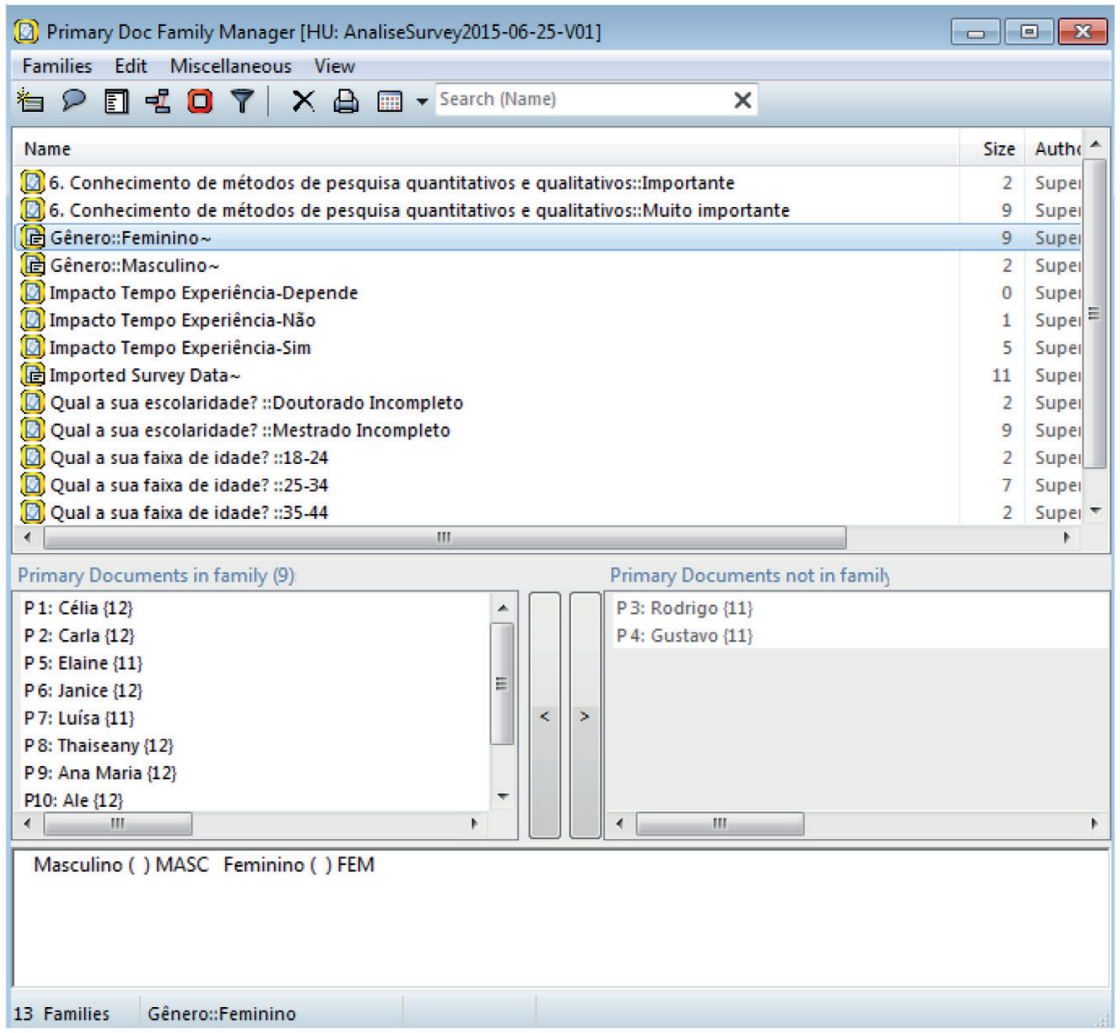

Fonte: acervo pessoal.

Os objetos utilizados no primeiro ciclo de codificação, também podem estar presentes no segundo ciclo de codificação, que é o momento no qual o pesquisador buscará desenvolver uma direção categorial, temática, conceitual ou teórica da codificação realizada durante o primeiro ciclo de codificação (SALDAÑA, 2016). Para isso, o pesquisador realiza novas organizações com os códigos, reanalisando os dados, podendo criar novas famílias e códigos. 
Para auxiliar esse processo, o ATLAS.ti oferece seis ferramentas de análise, sendo três quantitativas: contador de palavras (Figura 9), tabela de coocorrências (LUPEPSO; MEYER; VOSGERAU, 2016), tabela de codificação de documentos primários. E oferece três ferramentas qualitativas: o explorador de coocorrências; ferramentas de questionamentos (Query) e supercódigos.

Figura 9 - Ferramentas de análise

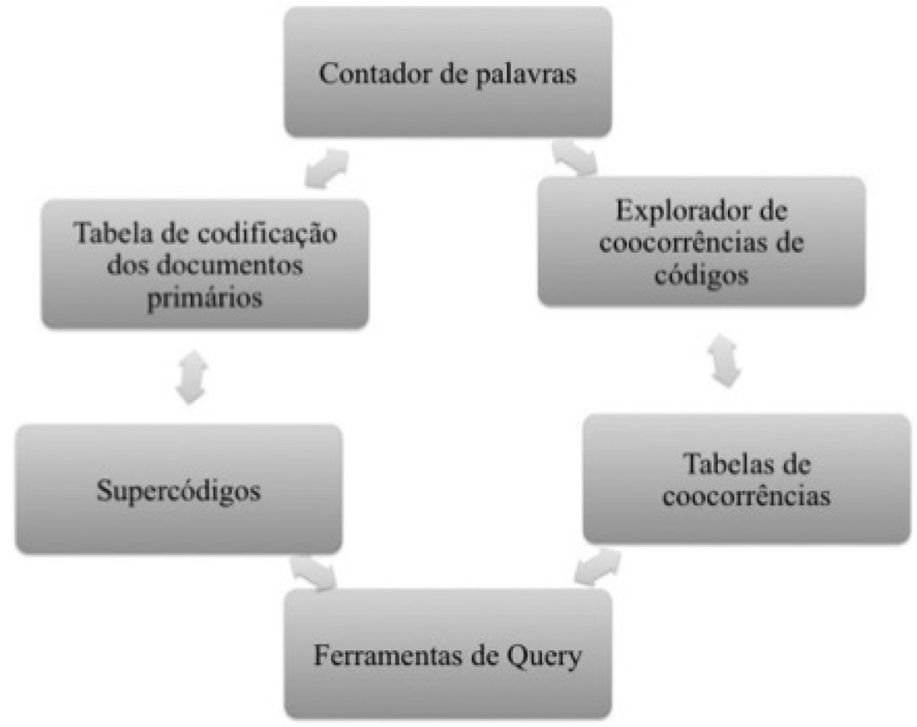

Fonte: Contreras (2015).

O uso dessas ferramentas não ocorre de forma sequencial, contudo, apenas o contador de palavras não depende de codificação prévia e pode auxiliar, em alguns casos, na tarefa de codificação, durante o primeiro ciclo de codificação, conforme foi utilizado por Pasinato (2011) e Rossari (2012) e tem por finalidade localizar a incidência de palavras relacionadas a tecnologias digitais em documentos oficiais de educação, para então proceder uma codificação automática dos extratos localizados. 
Pela explanação apresentada observa-se que o software para análise de dados qualitativos oferece um grande número de recursos, que combinados podem contemplar infinitos métodos para análise de dados qualitativos. No entanto, isso implica conhecimentos sobre esses métodos e domínio do uso dos recursos impondo novos desafios ao pesquisador em processo de formação.

\section{Desafios para utilização do software na análise de dados qualitativos}

Nos relato $s$ dos pesquisadores na palestra apresentada no XII EDUCERE fica evidente que consideram inúmeras as vantagens de terem utilizado o software em suas pesquisas (POCRIFKA, 2012; SIMONIAN, 2012; SIEVERT, 2013; FERREIRA, 2015; LUPEPSO; VOSGERAU; MEYER, 2016). Contudo, também foram unânimes em afirmar que a adoção do software impõe novos desafios, diferentes dos enfrentados pelos colegas que aplicaram o método tradicional de bricolagem e não utilizaram o programa na realização da análise.

Com o intuito de interligar a coleta e a análise, e dar suporte a uma primeira experiência com uso de software de análise de dados qualitativos, Meyer (2010) utilizou o diário de bordo nas duas etapas (Figura 10). Porém com ênfase no registro da história de sua análise, descrevendo cada uma das decisões tomadas e que definiram o seu plano de análise de dados, integrando os objetivos específicos, as observações relativas à aplicação dos instrumentos e até mesmo dúvidas acerca do uso do software de análise de dados qualitativos. A opção pelo diário de bordo surgiu da inexperiência e a consequente insegurança em relação ao processo e ao programa, assim como na tentativa de assegurar o rigor científico, além de subsidiar a redação da metodologia no relatório final da pesquisa. 
Figura 10 - llustra uso de diário de bordo na análise de dados

Diário de Bordo:

Dia $1^{\circ}$ de maio de 2009

Criação de códigos para PERFIL.

Comecei pelos que considerei essenciais para o propósito da análise que são função e tempo de atuação na Prefeitura de Curitiba como professor.

Diferenciei com letras maiúsculas o que é mais geral PERFIL e deixei padrão em seguida, complementando com Função ou Tempo atuação

Terei ao menos outros três campos principais, são eles, LEITURA, EXPECTATIVA e CONHECIMENTO. De acordo com os objetivos específicos da dissertação que estão relacionados a este instrumento de coleta de dados (questionário 1).

Em relação a LEITURA, podemos identificar:

Se tem Apreço pelo ato de ler,

Se teve Estímulo para o hábito de leitura,

Qual o Motivo pelo qual lê,

Em relação ao CONHECIMENTO, podemos identificar:

Identifica Modo de conhecimento do projeto

Sobre a Definição de jornal eletrônico escolar,

Sobre os Beneficios que aponta para o jornal eletrônico escolar,

Em relação a EXPECTATIVA, podemos identificar:

(Há questões fechadas que não sei como lidar)

Dúvida 1: Como codificar questőes fechadas? (sim e não)

Dúvida 2: Se faz marcação em uma mesma linha, fica vermelho? Ver

LEITURA Estímulo, LEITURA Apreço no sujeito 1.

Dúvida 3: A fonte do arquivo de origem independe ou precisa ser padronizada?

Dúvida 4: Por que na minha tela não fica em destaque a citação?

Dúvida 5: Não consigo perceber se a codificação que eu fiz é suficiente para relacionar o perfil de leitor do sujeito com o suas impressones sobre o jornal eletrônico, ou se deveria classificar de forma mais aprofundada por exemplo a definição se devo categorizar de maneira mais especifica. As duas alterações que eu fiz nos arquivos de origem, cliquei COMMIT CHANGES.

Fonte: Acervo próprio (2009)

O diário também registra o passo a passo do que está sendo realizado, permitindo que no período seguinte, o pesquisador saiba exatamente qual tarefa estava realizando a fim de que possa organizar as 
atividades a serem realizadas na empreitada a ser iniciada. Evidencia-se também um uso do diário de bordo tanto na superação de dúvidas operacionais sobre o programa, quanto na reflexão sobre a análise. O pesquisador descreve seus pensamentos e suas ideias, com a finalidade de, por meio do autoestudo, conseguir elucidar as questões que permeiam o processo de tomada de decisão na análise de dados e recorrer à pesquisa bibliográfica (Figura 11).

Figura 11 - Ilustra uso de diário de bordo na análise de dados

\section{FUI ATÉ O SUJEITO 7 Dia 4 de maio de 2009}

Inclui código LEITURA Sem apoio e LEITURA Dificuldade

Gostaria de incluir um comentário sobre o sujeito 9 , pois fica evidente que ele desconhece o projeto, mas não sabia se opção melhor era coments ou meme, então inseri no memo.

Dúvida: $\mathrm{E}$ quando determinado sujeito não apresenta um item da codificação, eu preciso sempre inserir meme, ou isso fica evidente na análise final? Como devo proceder?

Dúvida 2: Sinto que a codificação de definição precisa ser melhorada pois da forma com que está não leva a nenhuma análise, só aponta onde ela se encontra, mas não consegui ainda pensar em uma forma de melhorar esta codificação. Idem para codificação de motivos.

Sujeito 12 apresenta uma definição relacionada não aos benefícios, mas ao futuro profissional dos estudantes.

\section{7 de maio de 2009}

Dividir o código de estímulo de leitura pela época em que o estímulo foi realizado. Assim, a citação não ficará longa e não precisará conter o número da pergunta.

Idéia para dividir novamente codificação definição - em relação à natureza da definição. Se está atrelada à funcionalidade (para que serve), se está atrelada aos benefícios (o que proporciona de positivo), se é apenas descritiva.

Dividi o código de LEITURA ESTímULO e de CONHECIMENTO DEFINIÇÃO. Fonte: Acervo próprio (2009) 
A Figura 11 também evidencia que os memos, que serão mencionados a seguir, não necessariamente substituem a utilização do diário de bordo. Apesar de serem anotações ou comentários empreendidos no decorrer da análise, eles atuam como prévias reflexões preliminares que podem conduzir análise dos dados. Entretanto, os memos não têm um uso operacional, portanto, não atuam para demarcar as atividades realizadas, os problemas ou as dúvidas.

Outro desafio se refere ao manuseio de ferramentas Windows, complementares na etapa de preparação de dados, como no caso do Word, Excel (para utilização do recurso de importação de surveys) e mesmo o cuidado na organização de pastas. Como alguns deles (POCRIFKA, 2012; SIMONIAN, 2012) utilizaram a versão 6.0 do software existiam cuidados especiais a serem tomados com a nomenclatura de arquivos, pastas e ainda com a localização virtual dos documentos primários, o que os obrigava a ter um domínio maior do Windows Explorer, o não é muito comum em algumas áreas das Ciências Humanas e levavam, nessa época, alguns alunos a desistirem da utilização, por não conseguirem organizar e localizar seus dados dentro do computador.

Essas dificuldades foram resolvidas na versão 7.0, ao se acoplar as fontes de dados (primary documents) na própria biblioteca do projeto, portanto Sievert (2013), Ferreira (2015) e Lupepso, Vosgerau e Meyer (2016) não compartilharam da mesma ansiedade. Contudo, ainda existem softwares cuja fonte de dados não fica dentro do projeto e implicam um cuidado maior na criação e movimentação de pastas, elemento este a ser considerado ao se escolher um software para análise de dados.

Ainda na fase de preparação, Ferreira (2015) apresenta uma contribuição fundamental na escolha da plataforma a ser utilizada. $\mathrm{O}$ autor detinha certa experiência no uso do software ATLAS.ti em atividades acadêmicas empreendidas em disciplina. Ao ter o contato com o ATLAS.ti na versão MAC, adquiriu a licença de dois anos, mesmo sendo um usuário recente do Macbook. A justificativa foi a facilidade de manuseio, usabilidade e localização das ferramentas. 
Para a escolha das técnicas de análise Simonian (2012), Pocrifka (2012), Sievert (2013) e Ferreira (2015) em suas pesquisas utilizaram Bardin (2009), assim como grande parte dos estudos qualitativos brasileiros (SILVA et al., 2013). Os pesquisadores são unânimes ao afirmarem que muitas dificuldades que tiveram foram por falta de aprofundamento nos métodos e técnicas de análise de dados. Ferreira (2015), que tinha um grande volume de dados e passou por um exaustivo processo de codificação, relata que, em dado momento, precisou recorrer à elaboração de um protótipo por meio do processo de bricolagem para que com o aprofundamento da leitura de Bardin (2009) tomasse as decisões de agrupamentos e cruzamentos que seriam realizados com o software.

Complementarmente, Simonian (2012) e Pocrifka (2012) tendo utilizado amplamente o software em suas pesquisas de mestrado e retomando sua utilização para a pesquisa realizada no doutorado, garantem que o conhecimento aprofundado de técnicas de codificação, principalmente em formas de ciclos, como apresentadas por Saldaña (2016) podem oferecer mais alternativas para formulação de um plano prévio de análise de dados e uma compreensão melhor do que pode ser encontrado dentro do software.

\section{Considerações Finais}

A experiência vivida na palestra, ao trazer para os participantes do XII EDUCERE não apenas questões teóricas sobre as vantagens e desvantagens do uso do ATLAS.ti, mas a partilha de estudantes de Pós-graduação que desafiaram a si mesmos buscando aprimorar a qualidade de sua pesquisa, foi enriquecedora diante da oitiva dos anseios, dúvidas, desafios e sucessos. Também não se encerrou nas sugestões recebidas pela plateia que podem gerar novos caminhos a serem traçados nas pesquisas, mas especialmente em relação à continuidade do trabalho como formadora de novos pesquisadores qualitativos.

Pudemos confirmar nossas conjecturas ao longo desses 15 anos ensinando e aprendendo sobre o processo de análise de dados qualitativa, 
que, antes de tudo, o pesquisador precisa compreender e ser capaz de elaborar um plano de análise de dados que o auxilie a responder as questões e subquestões propostas em sua pesquisa, para então buscar no software as alternativas oferecidas para que possa aplicar o caminho previamente planejado. Grande parte da desistência dessa utilização não ocorre por barreiras tecnológicas, mas sim por dificuldades conceituais que não permitem ao estudante-pesquisador em formação visualizar as possiblidades oferecidas pelos softwares.

\section{Referências}

BARDIN, L. Análise de conteúdo (L. De A. Rego \& A. Pinheiro, Trads.). Lisboa: Edições 70, 1977. (Obra original publicada em 1977)

BARDIN, L. Análise de conteúdo (L. de A. Rego \& A. Pinheiro, Trads.). Lisboa: Edições 70, 2006. (Obra original publicada em 1977)

BARDIN, L. Análise de conteúdo. Edição revista e atualizada. Lisboa: Editora 70, 2009.

BUCHI, R. de F. Relações entre comunidades de prática e comunidades de aprendizagem. 2006. 127 f. Dissertação (Mestrado) - Pontifícia Universidade Católica do Paraná, Curitiba, 2006 Disponível em: <http://www.biblioteca.pucpr.br/tede/ tde_busca/arquivo.php?codArquivo=549>. Acesso em: 10 jan. 2016.

CONTRERAS, R. B. Qualitative data analysis with Atlas.ti 7 windows: introduction. Qualitative Data Analysis with ATLAS.ti 7 Windows Trainning. Apresentação PowerPoint utilizada no curso Qualitative Data Analysis with ATLAS.ti 7 Windows, Chigago, 10 a 12 mai, 2015.

CRESWELL, J. Research design: Qualitative, quantitative and mixed methods approaches (2nd ed.). Thousand Oaks, CA: SAGE Publications, 2003.

CRESWELL, J. W. Qualitative Inquiry and Research Design: Choosing Among Five Approaches. Los Angeles, CA: SAGE Publications, Inc, 2013. 
DENSIN, N. K.; LINCOLN, Y. S. Introduction: The Discipline and Practice of qualitative research. The Sage Handbook of Qualitative Research (4th ed., pp. 1-19). Thousand Oaks, CA: SAGE Publications, Inc, 2011.

ERCIKAN, K.; ROTH, W. M. What good is polarizing research into qualitative and quantittive? Educational Researcher. 35 (5), 14-23.

FERREIRA, A. B. de H. Novo Dicionário Aurélio da Língua Portuguesa. 4a. edição. Edição Eletrônica. Curitiba: Positivo Informática, 2009.

FERREIRA, J. de L. Formação continuada online para o desenvolvimento profissional dos professores que atuam no atendimento pedagógico ao escolar em tratamento de saúde. 2015. 341 p. Tese (Doutorado) - Pontifícia Universidade Católica do Paraná, Curitiba, 2015 Disponível em: <http://www.biblioteca.pucpr.br/tede/ tde_busca/arquivo.php?codArquivo=3426>. Acesso em: 9 maio 2016.

LEEDY, P.; ORMROD, J. Practical research: planning and design (7th ed.). Upper Saddle River, NJ: Merrill Prentice Hall. Thousand Oaks: SAGE Publications, 2001.

LUPEPSO, M.; MEYER. P.; VOSGERAU, D. S. R. Recursos Educacionais Abertos: potencialidades e desafios no Ensino Superior. Em avaliação.

MACHADO, M. M. O diário de bordo como ferramenta fenomenológica para o pesquisador em artes cênicas. Sala Preta, Vol. 2, 2016. Disponível em: < http:// www.revistas.usp.br/salapreta/article/view/57101 >. Acesso em 01 dez 2015

MACMILLAN, K.; KOENIG, T. The Wow Factor: preconceptions and expectations for data analysis software in qualitative research. Social Science Computer Review, Vol. 22 No. 2, Summer, 2004, 179-186.

MEYER, P. Possibilidades e limites na utilização do jornal eletrônico no processo de ensino-aprendizagem. 2010. 207 f. Dissertação (Mestrado) - Pontifícia Universidade Católica do Paraná, Curitiba, 2010. Disponível em: < http://www.biblioteca.pucpr.br/tede/tde_busca/arquivo.php?codArquivo=1679 >. Acesso em: 10 fev. 2015. MILES, M.B.; HUBERMAN, A. M. Qualitative Data Analysis. 2a.ed. London: Sage Publications, 1994. 
PASINATO, N. M. B. Proposta de indicadores para avaliação dos estágios de integração das TIC na prática pedagógica do professor. 2011. 138 f. Dissertação (Mestrado) - Pontifícia Universidade Católica do Paraná, Curitiba, 2011. Disponível em: <http://www.biblioteca.pucpr.br/tede/tde_busca/arquivo. php? codArquivo=2124 >. Acesso em: 12 mar. 2016.

POCRIFKA, D. H. Inclusão digital nas políticas públicas para formação de professores em Pernambuco. 2012. 181 f. Dissertação (Mestrado) - Universidade Federal de Pernambuco, 2012. Disponível em: <http://repositorio.ufpe.br/handle/123456789/12604>. Acesso em: 10 fev. 2015.

ROSSARI, M. A integração das tecnologias nos projetos políticos-pedagógicos: realidades e desafios. 2012. 215 f Dissertação (Mestrado) - Pontifícia Universidade Católica do Paraná, Curitiba, 2012, Disponível em: <http://www.biblioteca.pucpr.br/tede/tde_busca/arquivo.php?codArquivo=2530 >. Acesso em: 10 mar. 2016.

SALDAÑA, J. The Coding Manual for Qualitative Researchers. 2a . edição. Los Angeles: SAGE Publications, Inc, 2009.

SALDAÑA, J. The Coding Manual for Qualitative Researchers. 3a. edição. Los Angeles: SAGE Publications, Inc, 2016.

SIEVERT, G. Formação online para professores que atuam com escolares em tratamento de saúde. 2013. 144 f. Dissertação (Mestrado) - Pontifícia Universidade Católica do Paraná, Curitiba, 2013. Disponível em: <http://www.biblioteca.pucpr.br/tede/tde_busca/arquivo.php?codArquivo=2672>. Acesso em: 10 fev. 2015.

SILVA, A. H.; MOURA, G. L. De; CUNHA, D. E.; FIGUEIRA; K. K.; HORBE, T. A. N; GASPARY, E. Análise de conteúdo: fazemos o que dizemos? Um levantamento de estudos que dizem adotar a técnica. Anais... IV Encontro de Ensino e Pesquisa em Administração e Contabilidade, Brasília/DF, 3 a 5 de novembro de 2013. Disponível em: <http://www.posemcomunicacaodigital.com.br/wp-content/files/biblioteca/Analisedeconteudofazemosoquedizemos.pdf $\rangle$. Acesso em: 12 mar. 2016. 
SILVA, R. A. da. A mediação pedagógica em ambientes virtuais de aprendizagem: a formação continuada de professores do ensino fundamental. 2010. [175] f. Dissertação (Mestrado) - Pontifícia Universidade Católica do Paraná, Curitiba, 2010 Disponível em: <http://www.biblioteca.pucpr.br/tede/tde_busca/arquivo. php?codArquivo=1878>. Acesso em: 10 mar. 2016.

SIMONIAN, M. Formação continuada em ambiente virtual de aprendizagem: Elementos Reveladores da Experiência De Professores da Educação Básica. 2009. 162 f. Dissertação (Mestrado) - Universidade Federal do Paraná, 2009. Disponível em: 〈http://www.ppge.ufpr.br/teses/M09_simonian.pdf〉. Acesso em: 20 jul. 2016.

TAROZZI, M. O que é a grounded theory? Metodologia de pesquisa e de teoria fundamentada nos dados. Petrópolis, RJ: Vozes, 2011.

TESCH, R. Qualitative Research: Analysis Types and Software Tools. New York, Philadelphia; London: The Falmer Press, 1990.

WALTER, S. A.; BACH, T. M. Adeus papel, marca-textos, tesoura e cola: inovando o processo de análise de conteúdo por meio do ATLAS.ti. Administração: Ensino e Pesquisa, [S.1.], v. 16, n. 2, p. 275-308, jun. 2015. ISSN 2358-0917. Disponível em: 〈https://raep.emnuvens.com.br/raep/article/view/236>. Acesso em: 12 jul. 2016. doi:http://dx.doi.org/10.13058/raep.2015.v16n2.236.

Recebido: 15/08/2016

Received: 08/15/2016

Aprovado: 10/09/2016 Approved: 09/10/2016 
